\title{
Cell Hydrogen Oxidation
}

\author{
Thiyagarajan babu ${ }^{1}$, Chidambaram ${ }^{2}$
}

\begin{abstract}
Every individual's 75 to 100 trillion $\left(10^{14}\right)$ living cells fight with the millions of harmful radicals which were unstable molecules lacking one or more electrons and compensate by damaging healthy cells. Human body posses millions of glands all over the body to provide specialized products like proteins, fatty acids, hormones etc., to take care of any irregularity and inconsistency in the human system. Indeed the specialized glands like lachrymal gland, sweat gland, salivary glands etc., secrete only the essential compounds and those compounds reach cells associating with cellular water to protect the respective organs. The water every human being consumed was converted as cellular water (reduced surface tension and increased wetting ability) at standard ambient temperature and pressure and reverse was not possible in the cell system. The objective of this paper was that "No water molecules were externally released from human skin or any part of human cells except through kidney system." For example watery fluid formation inside the mouth involved different chemical reaction and it mixed with 'salivary compounds' forming 'saliva' contrary to existing saliva chemistry. The cell Hydrogen oxidation process was unknown due to the fact tears, saliva, nasal fluid and perspiration had the traces of compounds of respective glands and literatures cited they were the products of respective glands misleading the entire scientific community till date "My review is going to clear all the misconception and cell Hydrogen oxidation (redox potential balance) is explained more appropriately in this paper.
\end{abstract}

Key words: - Radicals, Glands, Molecules, Dehydration, Oxidation, Hyperhidrosis.

Accepted Date: 15 June 2013

\section{Introduction}

Hydrogen and Oxygen create all the energy required for heart, brain and liver cells in animals in the form of glucose and fat and surprisingly Oxygen burns Hydrogen in order to produce $\mathrm{H}_{2} \mathrm{O}$ and not $\mathrm{H}_{2} \mathrm{O}_{2}$ (sometimes damage cells). According to WHO (World Health Organization) definition - life is a continuous upgrading of amino acids in living organism through water and any starvation leads to acidic environment in the body making one to feel unhealthy. Sufficient amount of free electron (H-) in the human blood was the prerequisite for normal biological responses associated with the system or else human system find very difficult to cope up with the numerous chemical process required for the normal function leading to cell damage due to toxins (Source: http://www.medusmaize.lv).

Harmful radicals were easily formed in an acidic environment and they encourage solar radiation, exercise stress, passive smoking, and air pollution. Human life and cells were greatly influenced by the environmental signals and genes were not helpful in correcting biological responses like dehydration but surprisingly modified in response to life experiences and stimulate cells. Hence human beings are drinking water and 0.003 per cent Earth's fresh water is found within biological bodies. ${ }^{(\mathbf{1})}$ In a changed biological environment (malnutrition, polluted environment and stress) disease causing viruses, bacteria, fungi and parasites thrive more comfortable in more acidic condition; hence many glands secretes specialized compounds to protect the organs. It may be noted that water molecules neither directly support with any calories nor endow with nutrients but with water-based vulnerability where as approximately one billion people still lack access to safe water. ${ }^{(2)(3)}$

Infant's organism has more negatively charged hydrogen ions $(\mathrm{H}-)$, which neutralize harmful radicals and organize a normal biological reaction process. Human body cells had to maintain certain redox potential of different cell organelles such as blood, saliva, cellular solution, urine etc., In case of abnormality the cells had to spend their electric energy, which, actually, is the food transformational energy. Thus, in order to save organism energy, it is extremely important to use qualitative water. If you store more and more toxins in your body system then cell membrane had to spend their electric energy, which, actually available for food transformation energy being utilized to maintain blood, saliva etc in millivolts (Redox potential). This leads to less electric energy spent for food transformation and start all body complications like essential compounds and molecules disassociation, acidity, bacterial and viral growth proliferation and diseases, hence water consumption is obligatory.

Dehydration was avoided through water consumption and the human body contains approximately 55 per cent to 78 per cent water molecules depending on the level of body activity, temperature, humidity, body 
size and on other factors. ${ }^{(4)}$ There is no scientific base to advise any individual to drink eight glasses of water every day. ${ }^{(5)}$ And water in no way directly help in reducing human body weight and stomach upset (constipation) ${ }^{(6)}$ or directly released from any part of human cells but through oxidation. ${ }^{(8)}$ One milliliter for each calorie of food is the standard recommended for diverse persons and by and large, concerning twenty per cent of water ingestion come up to from food, at the same time as the rest comes from drinking water and beverages. ${ }^{[7]}$ Oxidation of Hydrogen molecules from the body cells would be one of the great concerns unknown till date. The true mechanisms were explained contradicting age old theories and beliefs with undeniable evidences.

\section{Glands}

Human beings are having two to four million glands spread around face, back, armpit, palm, skin, feet sole and groins to tackle various system problems and they range from 100 to 600 in number per square centimeter of skin.

According to Wikipedia, "A gland is an organ in an animal's body that synthesizes a substance (it can be understood: no tears and sweat or any external watery fluid secretions) for release often into the bloodstream (endocrine gland) or into body cavities or its' outer surface (exocrine gland) through ducts. Any blockage of those compounds in their respective ducts due to lack of smooth lubrication and transport through cellular water till epidermis leads to change in redox potential (abnormal symptoms) in the human system and side effects such as prickly heat in case of sweat glands. Some unexplained chemistry till date happens in the cells to form water fluid and to drain out the toxins from the skin pores externally due to pressure in every possible cell.

(A) Lachrymal Gland

(i) Types of tears :-

Eyes were continuously kept moist by basal tears found in the cornea region and the lachrymal fluid was associated with lipids, lactoferin, mucin, lacritin, glucose, urea, sodium and potassium providing nourishment to eyes and lysozyme which provides protection from bacteria. Reflex tears are formed when the external agents like bright light, tear gas, chilly pungency and other irritants like vomiting, painful coughing and yawning triggers TRP path in the ophthalmic nerve. ${ }^{(9)}$ Crying was due to physical pain, and positive (humor, laughter, happiness) as well as negative (anger, fear, sadness) emotional issues and tears secrete sometimes uncontrollably having traces of different chemical compounds. They contain more of enzymes like prolactin, adrenocorticotropic hormone and the amino acid leucine enkephalin (a natural painkiller) than first and second type of tears. It is further reported that the parasympathetic branch of the autonomic nervous system controls the lachrymal glands via the neurotransmitter acetylcholine through both the nicotinic and muscarinic receptors. When these receptors are activated, the lachrymal gland is stimulated to produce tears as per literature ${ }^{(\mathbf{1 2})}$ where as my results proved that lachrymal glands were actually stimulated to produce different compounds required for the protection of eyes and the cellular solution provided lubrication, wetting property and transportation making continuous tear film uninterrupted due to any blink and those glands eventually had no connection with the external tears formation. ${ }^{(8)}$

The nature of different types of tears formation as explained above had a psychic influence and typical brain actions and glands' role must be remote as per my findings..

\section{(ii) Tears Mechanism:-}

When human eyes were subjected for pain or involved with emotional disturbances, then former pressurized the cells in around and the eyes while the latter pressurized all the human body cells indirectly dislocating compounds of the cells. The human stress and emotions obviously affects redox potential of the organelles associated with eyes and tears are the one of the ways of balancing the same to avoid any damage to eye vision. Among the various compounds Hydrogen being the lightest with least survival time (less than a pico-second: $0.000000000001 \mathrm{sec}$.) gets the maximum pressure and this triggers the release of millions of lightest Hydrogen molecules like bullets from different tissues in search of an exit leading to oxidation of cell Hydrogen molecules. This was the play of the brain and it signals the powerful neurotransmitters to release the Hydrogen molecules in order to balance redox potentials of organelles and avoid any collision of two Hydrogen nucleuses due to cells' high pressure and temperature which was the principle of "Hydrogen Bomb." Otherwise the cells would accumulate more and more of Hydrogen molecules leading to any type of nucleus fusion, bulging and rupture of cells, there by disintegrating all cell content leading to the death of numerous cells ultimately entire body cells.

The eye lids were having specialized pores around the edges facilitating release of Hydrogen molecules and a cloud of Hydrogen molecules were found above the "tear film." ${ }^{(8)}$ The chain reaction of oxidation of Hydrogen molecules continues and the tears start forming and the drops get bigger, their weight deforms them into typical tear shape and shedding due to gravitational force. Due to gravitational force the tear film and the 
tears start mixing and fall down as tears and this was speciously reported as tears from lachrymal glands by numerous literatures. ${ }^{(9)}$ Hence it may be understood that the lachrymal glands secrete the essential compounds required for the protection of eyes where as the emotional or otherwise tears were secreted outside the tear film due to oxidation of Hydrogen molecules indirectly balancing redox potentials of organelles and shed as tears differing in Hydrogen concentration from the cellular solution (Fig.1).

As per existing literatures lachrymal fluid was secreted by the lachrymal glands and the fluid reaches the region of eyeball and lids through ducts. When the eyes blink, the lachrymal fluid was spread across the surface of the eye. Further, the existing literatures attributed that secretions that bathe and lubricate human eyes were called as tears besides powerful emotions for instance sadness, happiness, laughing, yawning, shock and joy over and above pain of eyes might direct to an augmented secretion of tears. ${ }^{(9)}$

The above scientific facts were contrary to science as cleansing and lubrication of eyes were protection process involving specialized compounds of the glands where as the external secretions were watery and secreted due to emotional disturbances and pain in and around the cells concerned.

The watery fluid naturally had some traces of products secreted by the gland due to solvency of the fluid. It was not wise to call both as 'tears' in my scientific view, hence the mechanism associated with both formations were comprehensible. The tear film was formed combining the special compounds secreted by the specialized glands associated with cellular water to lubricate the cornea and conjunctiva, to provide anti-bacterial activity, to keep the cornea moist, to serve as a channel for the entry of polymorphonuclear leucocytes into the cornea to remove toxic substances from the ocular surface for different protection of the eyes. ${ }^{(10)}$ They were not "tears" but certain compounds of lachrymal glands formed a film along with the cellular solution called as "tear film" as the surface tension of

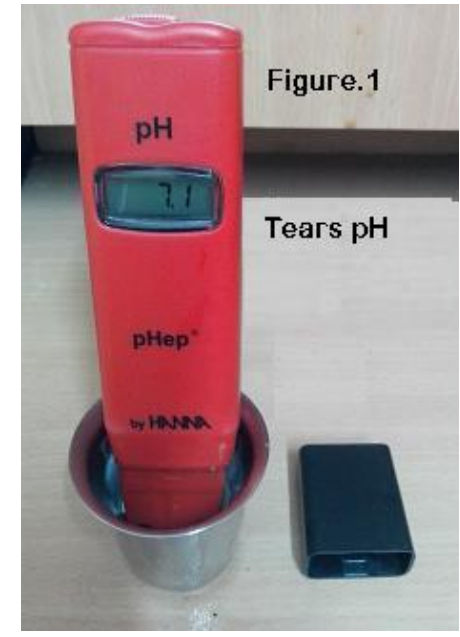
cellular water differed from "tears" secreted outside the eyes confirmed with pH meter (Fig.1). My study revealed that the lachrymal gland started secreting additional compounds as excess water molecule formation due to Hydrogen Oxidation would lead to washing away of tear film (dry eyes), hence crying had additional compounds to tolerate pain and protect any foreign body invasion. The autonomic nervous system controls the compounds of lachrymal glands via neurotransmitter acetylcholine through both the nicotinic and muscarinic receptors and not the tears and when these receptors are activated, the lachrymal gland is stimulated to produce required compounds but not tears.

My findings were supported by the fact having a thin tear film may prevent one's ability to wear contact lenses, as the amount of oxygen needed was higher than normal and contact lenses stop oxygen from entering the eye hence, eyes with thin tear film would dry out while wearing contact lenses. Special eye drops are available for contact lens wearers and certain types of contact lenses are designed to let more oxygen through to the eye (Source: Tears from Wikipedia, the free encyclopedia) which irrefutably supports my findings.

\section{(iii)Removal of Lachrymal Gland had no effect on Tears:-}

Any abnormality in the tear film leads to dry eyes affecting vision. ${ }^{(10)}$ A study on the change of tear film and lachrymal secretion after lachrymal gland tumor removal conducted at Eye and ENT Hospital of Fudan University, Shanghai 200031, China stated that complaints of dry eye increased and the mean tear break up time (BUT), reflex lachrymal secretion, and size of lachrymal lake were significantly reduced, but had returned to preoperative values at the sixth month visit. The result of the opposite eye control was the same as preoperative control and similar type of results were observed by Chinese. ${ }^{(11)}$ Further a study on tear production after unilateral removal of the main lachrymal gland in Squirrel Monkeys showed that total removal of the main lachrymal gland did not in itself lead to Keratoconjunctivitis sicca. However, the nature of neural control of the accessory glands was not yet clear. ${ }^{\mathbf{( 1 2})}$ Hence, tear film and lachrymal excretion changes after lachrymal gland tumor removal and not the tears and proving tears were produced by oxidation of Hydrogen molecules and balancing of redox potentials of organelles only.

\section{(iv) Mechanism of Nasal Fluid:-}


Literature quoted that any excess lachrymal fluid from eyes flew into the puncta due to capillary force and entered into the lachrymal canaliculi finally filling the lachrymal sac. This fluid further entered nasolacrimal duct which drains into the nasal cavity. An excess of tears, as with strong emotion, can thus cause the nose to run. And running nose which was collection of more fluid in the nasal cavity due to over crying, allergies, common cold or fever was a common phenomena and called as Rhinorrhea or rhinorrhoea. ${ }^{(20)}$

It was reported from Tears Wikipedia, the free encyclopedia in the Neural aspects that the trigeminal $V_{1}$ (fifth cranial) nerve boar the sensory pathway of the tear reflexes. When the trigeminal nerve was cut, tears from reflexes would stop, but not emotional tears. My study agreed upon the fact, except the scientific truth of lachrymal fluid formation which was due to cell Hydrogen molecules (from the cells around eyes) oxidization by atmospheric Oxygen. ${ }^{(8)}$

\section{(B) Sweat gland:-}

(i) Types of sweat glands:-

Sweating is a normal process regulating body temperature by cooling a hot body internally and externally thereby releasing toxins if present within body through skin involving sweat glands and they were the only source of cooling for body cells otherwise human body could invariably boil. A chain of coiled tubules and spiraled ducts carry sweat to the skin's surface. Some of the products like sexual musk or pheromones, secreted by sex hormones for sex attraction were carried and released by sweat glands. The estimated number of two million sweat glands is located in the dermis, or inner layer of skin, below the epidermis. In total more than 2.5 million sweat glands are found nearly everywhere on the human body, with several glands sited in armpits and soles of the feet, very few on the back, and none on the lips and nipples. ${ }^{(13)}$

There were two types of sweat glands namely Eccrine Gland and Appocrine Gland (most prominentcontain protein and fatty acids) present in great numbers on the palms, forehead and armpits. Those glands secrete different products at different stages of human life especially substances emitting unique odor. Eccrine Glands secrete (stress and physical exertion) essential products and cellular water combined with a small amount of potassium, high concentrations of chloride and sodium which were capable of cooling body cells. When eccrine glands secretions dry then, white salty patches on the cloth and body were obvious and balancing of redox potential of organelles remain behind all chemical reactions to save cells from any damage.

Appocrine glands (active during puberty, producing a "highly individual sexual scent") were present in armpits and groin region and secretes products like earwax and milk during breast feeding. Bacteria breakdown the fats secreted by those glands which were the reason for scent. But how the sweat gland products were brought out of skin needed more clarity in order to maintain the organelles redox potential. Literature quoted that a square inch, or 6.5 square centimeters, of skin holds hundreds of moisture-secreting sweat glands. ${ }^{\text {(13) }}$ Sweat seeps through the skin, evaporating while cooling the body surface down. Was that sweat mechanism which brought sweat gland products out of skin? No. The presence of different types of glands as explained above sensibly tells us that they were behind protecting skin surface and associated cells but how their products were carried and transported to needed cells required more explanation.

\section{(ii) Sweat Mechanism:-}

Sweating starts due to number of factors like hot sunshine, hard muscle work, individual situations affecting emotions, stress and irregular hormones production. Then the super computer, Brain, signals the neurotransmitters to trigger the sweat glands to produce products which would cool the cells leading to perspiration. The sweat glands produced compounds required to cool the body cells and they were carried to skin surface through different ducts carried by cellular solution ultimately maintaining the redox potential of different organelles present in every cell.

When the human beings underwent any type of tension due to unfavorable conditions like emotional issues, high temperatures and high relative humidity, the Hydrogen molecules were rushed out of cells and pass out through millions of pores as explained in the tears mechanism. Sweat was usually formed in a line over the skin as small droplets one after another as experienced by any one and that was the way the lightest Hydrogen molecules were released from skin pores and got oxidized. It may be noted that small sweat drops were formed gradually over the skin proportionate to the way Hydrogen molecules were released from cells during perspiration in order to maintain redox potential of the skin organelles. Each drop coalesces with adjoining sweat drop leading to increase in drop size and the volume of sweating. They were tested with the $\mathrm{pH}$ meter and ORP meter and significantly varied from cellular solution standard (Fig.2). Excessive secretion of sweat gland products could clog the sweat ducts and form red eruptions called as prickly heat on the skin surface causing itching and burning sensation. Once the gland is blocked, most commonly by skin oil and dead skin cells, the gland continues to secrete sweat products and oil which now had no outlet. Red eruptions were formed because the Hydrogen molecules could not get released from various cells though a range of pressures were subjected and sweat could not form lacking cell Hydrogen oxidization with the help of atmospheric Oxygen. ${ }^{(8)}$ 
The gland would then swell due to change in redox potential in the cell organelles leading to skin infection resulting in antibodies secretion to the site. The armpit was a very common place for sweat glands to get clogged, due to the folds of skin and the numerous hair follicles located in the region. ${ }^{(14)}$

When a person is not adapted to a high temperature climate, he can produce about a liter of sweat in an hour due to more and more Hydrogen molecules reaching the skin due to cell stress and got oxidized with atmospheric Oxygen. ${ }^{(8)}$ Extended stays in a hot environment affects redox potential of many cell organelles and can cause the body to increase its sweat production to the maximum capable volume of nearly 2 to 3 liters an hour depending on the Oxygen availability in that environment. As sweat fluid is watery and is a universal solvent it dissolves most of the products that come in contact with the skin while Hydrogen molecules were oxidized, losing excessive amounts of salt from the body through sweating which might lead to dehydration, so care should be taken and fluids should be replenished to balance $\mathrm{pH}$.

(iii) Sweat Gland Removal had no effect on Sweating:-

Is Hyperhidrosis (excess sweating) is due to high concentration of sweat glands? It further depend on the sympathetic nervous system helping transport of sweat gland products to skin surface. People with a past history of spinal cord injuries, peripheral neuropathies, probable brain lesions, an endocrine disease, a psychological disease, menopausal state or due to some medication experience excess sweat production in the area of the armpits (hyperhidrosis axillaris), in the hands and feet (hyperhidrosis manuum etpedum) or in the area of the head (hyperhidrosis facialis). ${ }^{(\mathbf{1 6})}$ In this case the theory 'sweating mechanism helps in maintaining body temperature' is disproved because the body maintains the temperature limits of inside and outside the body organs and redox potential limits of internal body fluids during excess sweating. This clearly supports my findings that sweat was produced externally due to cell Hydrogen oxidation by atmospheric Oxygen otherwise how body could maintain the body temperature and redox potential required for blood, urine, saliva, cellular solution etc., in the system.

Human body is filled with Hydrogen molecules (water and genes' Hydrogen-bonds) and obviously disassociate from any link due to its' lightest nature because of illnesses mentioned above. In such cases where excess sweating affects ones emotional level and daily activities, the menacing sweat glands can be removed. Some medicines like botulinumtoxin A ("botox") can give only temporary relief and by using sweat gland suction curettage, sweat glands were simply cleared out using suction, then roughened and their nervous linkage was disengaged. There were no complications but wound infections can occur and unwanted nerve damage might occur ${ }^{(16)}$ and how far sweat gland suction curettage like liposuction would help the patient was a big question? In my view it was one of the most dangerous processes complicating sweat gland mechanisms like fatty acids secretion and toxins removal. It was also reported that after sweat gland suction curettage, sweating conditions were reversed which proved that sweating was due to the oxidation of Hydrogen molecules balancing redox potential..

\section{(C) Salivary Gland:-}

(i) Types:-

Salivary glands are exocrine glands and secrete the essential compounds required for digestion of food but the liquid content in the mouth might have different chemistry. There are largest Parotid glands secretes saliva products through Stensen's ducts into the oral cavity; Submandibular glands secretes approximately 70 per cent of saliva products and enters the oral cavity via Wharton's ducts; Sublingual glands beneath the tongue secrete approximately 5\% of saliva products exit from 8-20 ducts; Minor salivary glands are present in oral cavity within the submucosa of the oral mocosa. The number of minor salivary glands runs to 800 to 1000 . They might be having many functions like lining the oral cavity with saliva and so; Circumvallated papillae of the tongue posses Von Ebner's glands secreting a product that begin lipid hydrolysis and they facilitate the perception of taste. ${ }^{(15,17)}$ The presence of different types of glands as explained above logically tells us that they were behind easing digestion of food but how their products were carried into oral cavity required more details.

(ii) Saliva secretion Mechanism:- 
The salivary gland secretes only the needed enzymes and compounds desirable for digestion of food and further they might mix with water molecules for lubrication and transport. Opening of mouth and breathing through nose and mouth facilitate Oxygen availability in the oral cavity in ample amount to oxidize the Hydrogen molecules released from different tissues due to mastication, Oxygen itself is a radical and how clever the cells of buccal cavity should be as Oxygen directly enters into throat and lungs. Hydrogen molecules help in maintaining the redox potential of saliva otherwise toxic Oxygen ultimately damage inner lining cells of buccal space, throat and lungs. hen the human beings were chewing the food, the tissues around the mouth and inside the oral cavity were invariably pressurized. Any type of tension due to favorite or unfavorable food calls human emotional mind set increasing body temperature, then the cell compounds were disturbed and the lightest and mean surviving Hydrogen molecules were rushed out of cells. That was one of the secret mechanisms of maintaining the redox potential of saliva. They were released into the oral cavity which was open to atmospheric Oxygen and those Hydrogen molecules were oxidized to form fluid namely "Saliva." Scientifically Oxygen burns Hydrogen molecules to make the saliva fluid (water molecules).

It might be clear that the liquid required for saliva was not secreted by the salivary gland but the essential compounds and enzymes (amylase, an enzyme that breaks down starch into maltose) associated with cellular solution needed for the saliva in order to fasten digestion. But various literatures ${ }^{(\mathbf{1 7})}$ were quoting that saliva was entirely a secretion of salivary glands which were disproved by my simple experiments. When the $\mathrm{pH}$ and redox of saliva were examined using $\mathrm{pH}$ meter and ORP (Oxidation Reduction Potential) meter respectively, they differed from cellular solution confirming source of the saliva (Fig.3). The salivary glands of insects secrete proteins for example silk or glues and fly salivary glands secrete products having polygene chromosomes and lubrication and needs entomological explanation.

\section{(iii) Salivary gland removal no effect on saliva:-}

Salivary gland (submandibular gland and parotid gland) and salivary duct stones are not uncommon and they had to be removed surgically to solve the issue. And patients found eating and drinking difficult for one or two days and recovered about one week time after salivary gland removal. The drain of some fluid (cellular solution) from wound was common. Some patients had some uncommon symptoms like swelling below jaw line and loss of taste and sensation. ${ }^{(17)}$

There were no complications after the removal of salivary gland but issues like infection, swelling, nerve damage, and build up of saliva were common and cured without treatment (source: http://www.cedarssinai.edu). In case of dogs hyper salivation was reported after the removal of salivary mucoceles which was due to Hydrogen Oxidation. ${ }^{(18)}$ The all complications were due to redox potential balancing and this supports that the watery fluid was added to salivary gland products only in the oral cavity through cell Hydrogen molecules Oxidation.

1.

(a) By disconnecting atmospheric Oxygen by just closing one's eyes tears can be reduced.

(b) A bike ride increases the tears or running nose: Eyes get contact with plenty of atmospheric Oxygen.

(c) Lighting fire or camp fire would consume atmospheric Oxygen and no place for tears but joy.

(d) Avoiding fresh air can reduce running nose as fresh air containing Oxygen was blocked.

\section{Saliva}

(a) Sleeping opened mouth leads to mouth watering and dry throat. Is it saliva? No.

(b) Anybody can avoid nuisance spitting by keeping mouth shut as Oxygen could not enter buccal space.

(c) Over talking produces excess saliva and watering hence close your mouth sometime to stop watering.

\section{Sweating}

(a) When you were in fresh air skin do not produce any sweat because Hydrogen molecules were intact in 
your cells and cell organelles redox potentials were not affected.

Oxygen suffocation leads to sweating because the cell Hydrogen molecules which were released due to cell pressure were oxidized exhausting available atmospheric Oxygen in the air in that environment ultimately fainting sometimes.

\section{Conclusion}

It is ironical to mention that the science had been teaching generation after generation that entire external watery secretion were directly released out of human cells as tears, perspiration or saliva owing to some of the specialized glands. Numerous literatures were the base for those incorrect facts and belief since civilization and many theories were supporting the same fact since centuries. I am to conclude that the lachrymal gland, sweat gland and salivary gland products are associated with cellular solution but the watery support was exclusively given by the cell Hydrogen molecules oxidation externally by atmospheric Oxygen in the process of balancing different cell organelles redox potentials. The fact that when these glands were infected or removed affects only the protection of eyes, skin and digestion (intestinal adaptation to food) respectively but watery fluid was always provided by Hydrogen oxidation as explained.

I put my maximum efforts in explaining mechanisms of tears, sweat, saliva and nasal fluid in this paper with the view to understand basic mechanisms of biological water in human body system than it was done in my "Noble Ganga Sweat-Tears Bomb Theory." And any secretion is under the control of nervous system e.g. sweating is the activity of the sympathetic nervous system found both sides of the spinal column and glands were not involved in it but in the production of proteins and other compounds.

I once again confirm that human cells do not release any amount of stable Oxygen from any part of body along with Hydrogen molecules to form water molecules. And it is interesting to mention that children and aged ${ }^{(19)(20)}$ salivate more than normal adults and it could be noted that the salivary glands were developing in case of children where as in aged people, the salivary gland function declines but not the saliva. That was all redox potential balancing scientific magic in the human system, All these prove that the tears, sweat and saliva secretions for the matter any external watery fluid formation on the human skin or body surface were the result of cell Hydrogen Oxidation and the glands had no role in those part.

\section{References}

[1]. Gleick, PH., Water in Crisis: A Guide to the World's Freshwater Resources. Table 2.1 "Water reserves on the earth."Oxford University Press. 1993; p 13.

[2]. Guyton, Arthur C. Textbook of Medical Physiology (5th ed.). Philadelphia: W.B. Saunders. 1976; p. 424. ISBN 0-7216-4393-0.

[3]. "MDG Report 2008". Retrieved 2010-07-25.

[4]. Re: What percentage of the human body is composed of water? Jeffrey Utz, M.D., The MadSci Network.

[5]. Drink at least eight glasses of water a day." Really? Is there scientific evidence for "8 $\times$ 8"? by Heinz Valdin, Department of Physiology, Dartmouth Medical School, Lebanon, New Hampshire.

[6]. Food and Nutrition Board, National Academy of Sciences. Recommended Dietary Allowances. National Research Council, Reprint and Circular Series, No. 122. 1945; pp. 3-18.

[7]. "Eye, human."Encyclopædia Britannica from Encyclopædia Britannica, 2006; Ultimate Reference Suite DVD 2009.

[8]. Thiyagarajan Babu, , Noble Ganga Tears-Sweat Bomb Theory, (IOSR-JNHS) e-ISSN: 2320-1959.p- ISSN: 2320-1940. 2013; 1 (3), 52-54.

[9]. Skorucak A. "The Science of Tears." ScienceIQ.com. Accessed September 29, 2006.

[10]. Holly FJ, Lemp MA, , Tear physiology and dry eyes, Surv Ophthalmol, 1977; Sep-Oct; 22 (2) : 69-87.

[11]. Zhang YQ, Qian J, Yuan YF, Xue K, Guo J, Wang XN. Tear film and lacrimal excretion changes after lacrimal gland tumor removal,Zhonghua Tan Ke Za Zhi, Jan; 2013; 49 (1) : 27-31.

[12]. Dmitri Y. Maitchouk, MD; Roger W. Beuerman, PhD; Toshihiko Ohta, MD; Michael Stern, PhD; Ray J. Varnell, PhD., Tear production after unilateral removal of the main lacrimal gland in Squirrel Monkeys, Arch Ophthalmol. $2000 ; 118$ (2): $246-252$. doi:10.1001/archopht.118.2.246.

[13]. Wilke, K, Keil, F.J., Wittern, KP. et al. Immunolabelling is essential for the differentiation of human axillary apoeccrine glands. J. Invest. Dermatol. 2004; 123, A93.

[14]. Kennedy, WR. Wendelschafer-Crabb, G, Brelje, TC. "Innervation and vasculature of human sweat glands: an immunohistochemistry-laser scanning confocal fluorescence microscopy study". The Journal of neuroscience: the official journal of the Society for Neuroscience 1994; 14 (11 pt. 2): 6825. ISSN 0270-6474.

[15]. Reisfeld, Rafael; Berliner, Karen I. "Evidence-Based Review of the Nonsurgical Management of Hyperhidrosis". Thoracic Surgery Clinics 2008; 18 (2): 157-66.doi:10.1016/ j.thorsurg. 01.004. PMID 18557589.

[16]. Lawrence CM, Lonsdale Eccles AA, , Selective sweat gland removal with minimal skin excision in the treatment of axillary hyperhidrosis: a retrospective clinical and histological review of 15 patients, Br J Dermatol. Jul 2006; 155 (1): $115-8$.

[17]. TEN CATE AR, "Oral histology. Development, structure, and function".1998, 2003, 2008 Edition=5thISBN=0-8151-2952-1.

[18]. Benjamino KP, Birchard SJ, Niles JD, Penrod KD, Pharyngeal mucoceles in dogs: 14 cases. JAAHA 2012; $48: 31-35$.

[19]. Yeh CK, Johnson DA, Dodds MW, Impact of aging on human salivary gland function: a community-based study, Aging (Milano), Oct $1998 ; 10(5): 421-8$.

[20]. Eileen Trigoboff; Kneisl, Carol Ren; Wilson, Holly Skodol. Contemporary psychiatric-mental health nursing. Upper Saddle River, N.J: Pearson/Prentice Hall. 2004; p. 274. ISBN 0-13-041582-0.

${ }^{1}$ Dr. Thiyagarajan Babu, Scientist ${ }^{2}$ Dr. N. Chidambaram, MD, Prof. Of Medicine, Principal, Rajah Muthiah Medical College \& Dean, Faculty of Medicine, Annamalai Univedsity, Annamalainagar-608 002 